\title{
Long-term memory in experiments and numerical simulations of hydrodynamic and magnetohydrodynamic turbulence
}

\author{
P. Mininni ${ }^{1,2}$, P. Dmitruk ${ }^{1}$, P. Odier ${ }^{3}$, J.-F. Pinton ${ }^{3}$, N. Plihon ${ }^{3}$, G. Verhille ${ }^{4}$, R. Volk ${ }^{3}$, M. Bourgoin ${ }^{5}$, \\ ${ }^{1}$ Departamento de Física, \\ Facultad de Ciencias Exactas y Naturales, \\ Universidad de Buenos Aires and IFIBA, \\ CONICET, Ciudad Universitaria, \\ 1428 Buenos Aires, Argentina. \\ ${ }^{2}$ Computational and Information Systems Laboratory, \\ NCAR, P.O. Box 3000, Boulder, \\ Colorado 8030\%-3000, USA. \\ ${ }^{3}$ Laboratoire de Physique - UMR 5672 \\ Ecole Normale Suprieure de Lyon / CNRS \\ 46 Allée d'Italie, 69007 Lyon, France \\ 4 Aix-Marseille Université, \\ IRPHE - UMR 7342, CNRS, Marseille, France \\ 5 Laboratoire des Ecoulements Géophysiques et Industriels - UMR 5519 \\ Université Joseph Fourier / Grenoble-INP / CNRS \\ BP53, 38041 Grenoble cedex 9.
}

(Dated: January 24, 2014)

\begin{abstract}
We analyze time series stemming from experiments and direct numerical simulations of hydrodynamic and magnetohydrodynamic turbulence. Simulations are done in periodic boxes, but with a volumetric forcing chosen to mimic the geometry of the flow in the experiments, the von Kármán swirling flow between two counter-rotating impellers. Parameters in the simulations are chosen to (within computational limitations) allow comparisons between the experiments and the numerical results. Conducting fluids are considered in all cases. Two different configurations are considered: a case with a weak externally imposed magnetic field, and a case with self-sustained magnetic fields. Evidence of long-term memory and $1 / f$ noise is observed in experiments and simulations, in the case with weak magnetic field associated with the hydrodynamic behavior of the shear layer in the von Kármán flow, and in the dynamo case associated with slow magnetohydrodynamic behavior of the large scale magnetic field.
\end{abstract}

\section{INTRODUCTION}

Since the development of hot-wire measurements in hydrodynamic turbulence in the laboratory, several reports of long-term memory in velocity time series (i.e., nonnegligible correlations in time scales much larger than the time scale associated with the energy containing eddies in the flow) can be found in the literature (see, e.g., [1, 2] for recent reports). The origin of such long-term correlations is unclear, as current knowledge of turbulence indicates that three-dimensional flows should transfer energy from the energy containing scale towards smaller scales (where the characteristic time scales are shorter). Therefore, no correlations at time scales longer than the energy containing scale are to be expected.

Evidence of long-term memory (or "long-range dependence") is often observed in the frequency spectrum of time series as a range of frequencies corresponding to $1 / f$ noise. By $1 / f$ noise (also often called "flicker" noise), it is usually meant that the power spectrum of the signal is of the form $E(f) \sim f^{-\alpha}$, where $f$ is the frequency, and with $\alpha$ loosely between 0.5 and 1.5 . The case with $\alpha=1$, which strictly speaking corresponds to $1 / f$ noise, is the case with equal energy per octave independent of the frequency. The range of frequencies in which this phenomenon often develops corresponds not only to fre- quencies smaller than the frequency associated with the energy containing eddies, but often also to frequencies that (if Taylor hypothesis are used) are associated with length scales much larger than the physical extension of the fluid. Therefore, such correlations cannot be associated with convective motions in the turbulent flow, and must be associated with long-term modulations in the system.

Observations of $1 / f$ fluctuations are not exclusive of hydrodynamic or magnetohydrodynamic (MHD) turbulence, and are widely found in natural and nonlinear systems [3]. As a result, they are often considered as a signature of scale invariant features of an underlying dynamical process. Time signals displaying $1 / f$ noise in their spectral density have been reported in electronics, tree growth, and human activities such as music and the stock market [4]. In conducting fluids and plasmas, $1 / f$ fluctuations have also been reported (see e.g., [5, 6] ). In the interplanetary magnetic field, its presence has been known for some time through analysis of time signals measured in situ near Earth's orbit [7-9]. Long-term behavior has also been found in time series of the geomagnetic field [10 12$]$, and in dynamo laboratory experiments 13 .

This ubiquity has motivated several works that attempted to identify general sources of $1 / f$ noise and 
long-term memory. Recent studies focused in the particular cases of hydrodynamic and MHD flows [6, 14] obtained $1 / f$ in MHD with and without a background magnetic field, but did not observe it in isotropic hydrodynamic flows. The modes giving the dominant contribution to the long-term correlations were identified to be modes with the largest available wavelength in the domain. Random isotropic forcing, with a short-time memory to give a single (unit) correlation time, was used to stir the flows in [6], while random initial conditions in ideal systems were considered in [14]. It was concluded that long-term memory is more easily seen in systems that develop inverse cascades, or that have non-local interactions [15]

In this work, we consider time series stemming from experiments and direct numerical simulations of hydrodynamic and MHD turbulence. Simulations are done in periodic boxes, but with a volumetric forcing chosen to mimic the geometry of the flow in the experiments. Parameters in the simulations are also chosen to (within computational limitations) allow comparisons between the experiments and the numerical results. Two different configurations are explored: the case with an externally imposed magnetic field (but with the magnetic field weak enough that can be considered a passive vector tracer and used to characterize the flow), and the case with selfsustained magnetic fields. The former case (corresponding to "induction," as the magnetic field fluctuations observed are induced by motions of the conducting flow) can be relevant for many liquid metal laboratory experiments and for industrial flows. The latter, corresponding to a magnetohydrodynamic dynamo, has implications for magnetic field generation in geophysics and astrophysics.

\section{EXPERIMENTS AND SIMULATIONS}

\section{A. Experiments}

Experimental data discussed in this article has been obtained in the VKG and VKS experimental setups (see Fig. 1). The VKG experiment is a von Kármán swirling flow of liquid Gallium, while VKS is a von Kármán flow of liquid Sodium. In both cases the von Kármán flow is generated in the gap between two counter-rotating impellers in a cylindrical vessel. This geometry has been extensively investigated in the past decade in the context of magnetic field self-generation by dynamo effect. Its interest in MHD studies has been motivated by the large scale topology of the generated flow which exhibits strong differential rotation and helicity.

The VKG experiment is mostly designed to investigate MHD mechanisms as induction processes (transport and deformation of magnetic field lines by the flow) and flow modification by an imposed magnetic field (via the Lorentz force acting on the moving conducting fluid). The reachable magnetic Reynolds number $R m=U L / \eta$ (which compares the magnetic induction effects to mag-

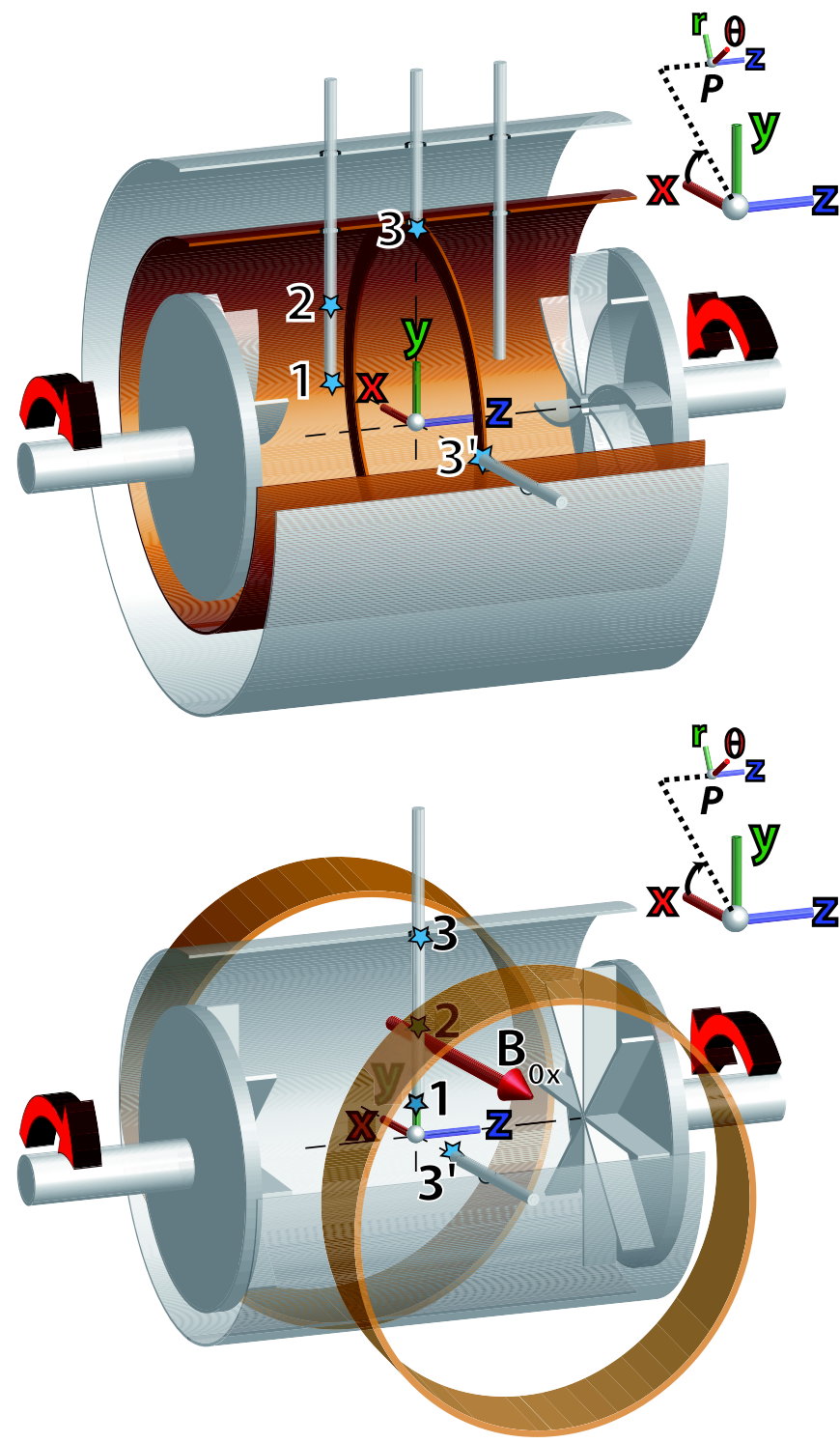

FIG. 1. (Color online) Sketch of the experimental setups. (a) VKS experiment drives a von Kármán swirling flow of liquid sodium in a cylindrical vessel. Data from the experiments reported here was obtained in a configuration with an inner coper shell and an annulus in the mid-plane. (b) VKG experiment drives a von Kármán flow of liquid gallium in a cylindrical vessel. A pair of lateral Helmholtz coils allows us to apply a transverse magnetic field. Positions of the measurement probes are indicated with the stars.

netic dissipation effects, with $U$ the typical velocity of the flow, $L$ its typical dimension and $\eta$ the magnetic diffusivity) accessible in VKG is of order unity so that no dynamo effect is expected to be observed in this experiment. On the other hand, the VKS facility has been designed and optimized for dynamo generation. It is twice as large as VKG, with an available mechanical power 15 times larger, and it uses liquid Sodium which is less resistive than Gallium. As a consequence, accessible magnetic Reynolds number in VKS is of the order of 50 . In 
2006 the first dynamo generation was observed in VKS when soft iron-impellers were used to drive the flow. Details of the VKG and VKS experiments can be found in 13, 16, 17.

As for all liquid metals, the magnetic Prandtl number (defined as the ratio of the kinematic viscosity $\nu$ of the fluid to its magnetic diffusivity $\eta, \mathrm{Pm}=\nu / \eta$ ) of Gallium and Sodium is very small $\left(\mathrm{Pm}^{\mathrm{Ga}}=1.4 \times 10^{-6}\right.$, $\left.\mathrm{Pm}^{N a}=6.2 \times 10^{-6}\right)$. As a consequence, flows with magnetic Reynolds number Rm of order unity or larger have a kinematic Reynolds number $\operatorname{Re}=U L / \nu=\mathrm{Rm} / \mathrm{Pm}$ exceeding $10^{5}$, hence operating in highly turbulent conditions. Magnetic processes in VKG and VKS experiments are therefore submitted to highly fluctuating turbulent conditions. To this respect, von Kármán flows are particularly interesting as they are known to present a wide hierarchy of scales of fluctuations. At intermediate and small scales, the flow behaves as traditional turbulence (although anisotropic), with for instance a classical $k^{-5 / 3}$ Kolmogorov spatial spectra. At larger scales the flow undergoes in many cases long-term dynamics, which in the absence of strong or self-generated magnetic fields, are mostly driven by hydrodynamic instabilities of the midplane shear layer [2, 18].

As already mentioned, the VKG experiment is meant to investigate magnetohydrodynamic induction processes with magnetic Reynolds number $\mathrm{Rm}<1$. To this purpose a pair of transverse coils is placed laterally to the experiment and allows to apply a transverse magnetic field $B_{0 x}$. When the applied magnetic field is sufficiently weak so that the interaction parameter $N=\mu_{0} L B_{0 x}^{2} / \eta \rho U$ remains low and of the order of $10^{-3}$, the imposed magnetic field can be considered as a passive vector, advected by the flow. Recent experiments [19] have shown that this approximation stops to be valid as soon as $N \geqslant N^{*} \sim 0.02$, in which case the flow itself is modified by the applied magnetic field. Only the situation in which the magnetic field is weak and acts as a passive vector will be considered here. In this case, the magnetic field can just be used as a way to measure and characterize the hydrodynamic flow.

The VKS experiment has several possible configurations. We will focus here on the geometry sketched in Fig. 11 (top), which has been extensively investigated and for which the main results have been reported in Ref. [13]. The flow is driven with iron impellers, such that a self-sustained dynamo magnetic field is observed above a magnetic Reynolds number threshold of the or$\operatorname{der} \mathrm{Rm}^{c} \approx 30$.

Accessible measurements in VKS and VKG are mainly magnetic, as well-resolved velocimetry is extremely difficult in liquid metals, though some kinematic measurements have been recently made accessible thanks to a local miniature Vivès probe [20]. The induced or selfsustained magnetic field is measured inside the flow by means of Hall probes. In the VKG experiments, measurements were accessible on a line along the vertical $O y$ axis in the mid-plane (see the numbered stars in the bot- tom panel of Fig. 11) few measurements along the $O x$ axis in the mid-plane are also available. In the VKS experiments, measurements were accessible at the locations indicated by stars in Fig. 1 (top).

\section{B. Numerical simulations}

The numerical simulations solve the equations for an incompressible flow in a three-dimensional periodic domain of side $2 \pi$. In the most general case (a conducting fluid with an externally imposed magnetic field of strength $\mathbf{B}_{0}$ ), the equations in dimensionless Alfvenic units read

$$
\begin{gathered}
\frac{\partial \mathbf{u}}{\partial t}+\mathbf{u} \cdot \nabla \mathbf{u}=-\nabla \mathcal{P}+\mathbf{j} \times\left(\mathbf{b}+\mathbf{B}_{0}\right)+\nu \nabla^{2} \mathbf{u}+\mathbf{F}, \\
\frac{\partial \mathbf{b}}{\partial t}+\mathbf{u} \cdot \nabla \mathbf{b}=\left(\mathbf{b}+\mathbf{B}_{0}\right) \cdot \nabla \mathbf{b}+\eta \nabla^{2} \mathbf{b} .
\end{gathered}
$$

In the incompressible case $\nabla \cdot \mathbf{u}=0$, and $\nabla \cdot \mathbf{b}=0$. Here, $\mathbf{u}$ is the velocity, $\mathbf{b}$ is the magnetic field, and $\mathbf{j}=\nabla \times \mathbf{b}$ is the current density. The pressure (normalized by the density) is $\mathcal{P}, \nu=4.7 \times 10^{-3}$ is the dimensionless kinematic viscosity, and $\eta$ the magnetic diffusivity, with $\eta=\nu / \mathrm{Pm}$. In the absence of an externally imposed magnetic field $\left(\mathbf{B}_{0}=0\right)$ these equations are the incompressible MHD equations often used to study the dynamo effect. When $\mathbf{B}_{0}=\mathbf{b}=0$ (or when the interaction parameter $N$ is small enough), Eq. (1) reduces to the Navier-Stokes equation for an incompressible hydrodynamic flow.

To mimic the geometry of the flow in the experiment, a Taylor-Green (TG) vortex is used as mechanical forcing

$$
\mathbf{F}=A_{0}\left[\begin{array}{c}
\sin \left(k_{0} x\right) \cos \left(k_{0} y\right) \cos \left(k_{0} z\right) \\
-\cos \left(k_{0} x\right) \sin \left(k_{0} y\right) \cos \left(k_{0} z\right) \\
0
\end{array}\right],
$$

where $A_{0}=0.41$ is the forcing amplitude, and $k_{0}=2$ is the forcing wavenumber. This forcing was shown before to give good agreement with von Kármán dynamo results [21, 22], while allowing simulations to be done in periodic boundaries and thus lowering computational costs. A sketch of the resulting flow in one TG cell can be seen in Fig. 2,

Equations (11) and (2) are solved pseudospectrally using a parallelized code $23-25]$ dealiased with the $2 / 3$ rule. Second-order Runge-Kutta is used to evolve the equations in time. Since long integrations are needed (in many cases simulations were continued for over 6000 turnover times), linear spatial resolution is $N_{\text {res }}=128$ in all cases. To compare with experiments, time series of the three Cartesian components of the velocity and the magnetic fields at the three points indicated in Fig. 2 are recorded with high cadence. Also, time series of the amplitude and phase of each Fourier mode in spectral space 


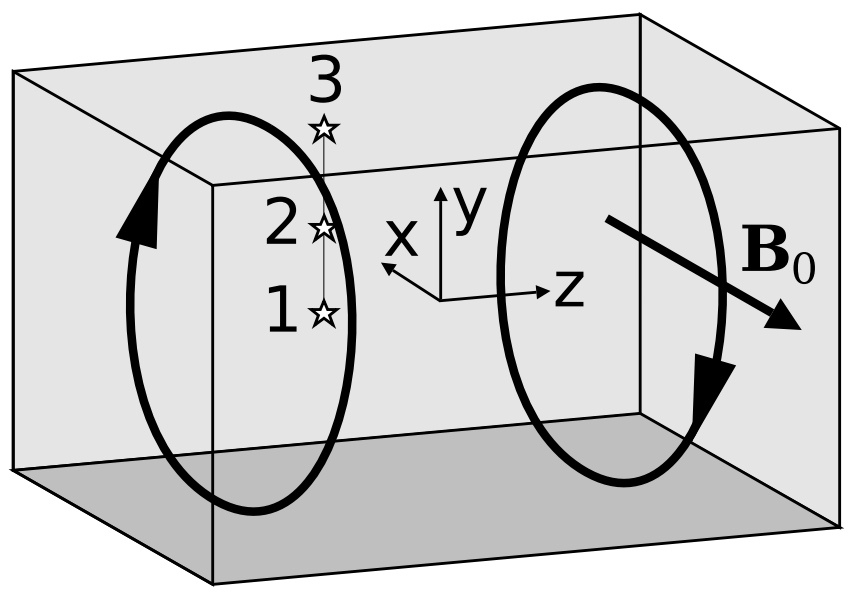

FIG. 2. Sketch of the numerical Taylor-Green cell, and position of the points where magnetic and velocity measurements are made (indicated with stars). The two large circular arrows represent the two counter-rotating Taylor-Green vortices.

are stored, to be able to identify the modes responsible for long-term behavior. Note that with the spatial resolution considered here, magnetic Reynolds numbers similar to the ones in the experiments are easily reproduced. However, the mechanical Reynolds numbers in the experiments are out of reach for even the largest resolutions we could attain in simulations, and those resolutions would preclude the long time integrations considered in this study.

The strategy used in the simulations is as follows. The mechanical force is turned on at $t=0$ from the flow at rest, and the Navier-Stokes equations (with the $\mathbf{b}$ and $\mathbf{B}_{0}$ fields set to zero) are advanced for over 6000 turnover times (a turbulent steady state is reached shortly after 10 turnover times). Time series of the velocity are then used for the analysis of $1 / f$ noise in hydrodynamic flows (as well as to compare with the equally long simulations with a weak imposed magnetic field). Later, the last state of the hydrodynamic flow is used to start two different sets of simulations: one in which a uniform magnetic field $\mathbf{B}_{0}=B_{0} \hat{y}$ is imposed, and one in which $\mathbf{B}_{0}=0$ and an initially small magnetic seed is amplified until an MHD steady regime is reached. The former is intended to mimic induction experiments with a transverse magnetic field in VKG, while the latter is intended to mimic dynamo experiments in VKS. In both cases the systems are integrated for over 6000 turnover times. The magnetic Prandtl number in induction simulations is $\mathrm{Pm}=0.05$ and the interaction parameter is adjusted by setting $B_{0}$ such that $N \approx 10^{-4}$. In dynamo simulations $\mathrm{Pm}=0.5$ as a larger magnetic Reynolds number is needed to sustain magnetic fields 21, 22]. The mechanical Reynolds number is fixed in all simulations, and is $\mathrm{Re} \approx 670$.
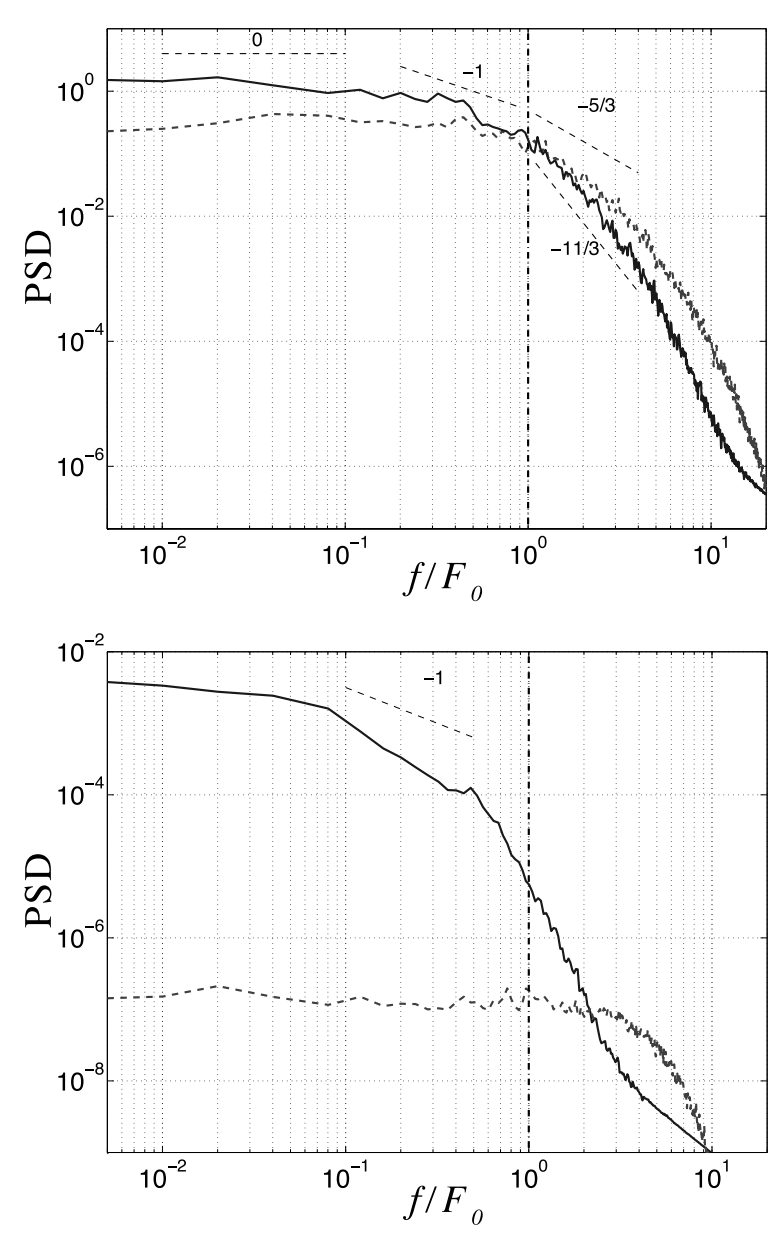

FIG. 3. Numerical simulations of Taylor-Green MHD induction. Top: Power spectrum of the temporal fluctuations of the axial velocity $u_{z}$ at point 1 (light gray dashed line) and of the axial induced magnetic field (dark gray solid line) in the numerical simulations at low magnetic Prandtl number $\left(\mathrm{Pm}=0.05\right.$ and low interaction parameter $N=4 \times 10^{-5}$. Frequency axis has been normalized by the forcing frequency $F_{0}=u_{r m s} / \pi$. The global energy level of the spectra has been arbitrarily set to make them coincide for $f=F_{0}$. Bottom: Power spectrum of the amplitude of Fourier mode $k=(1,0,0)$ of $u_{z}$ (dark gray solid line), and of Fourier mode $k=(10,10,10)$ of $u_{z}$ (light gray dashed line). In both panels, several slopes reported before in the VKS and VKG experiments are shown as references, although we are mostly interested in the behavior for $f<F_{0}$.

\section{HYDRODYNAMIC TURBULENCE AND LOW $N$ INDUCTION}

When the imposed magnetic field is weak enough that it can be considered a passive vector, time series of the velocity field measured in the experiments and simulations at any of the points indicated in Figs. 1 and 2 do not show clear long-term behavior or $1 / f$ noise, as it can be seen for instance from the dashed lines in Fig. 3 (top) and 4 which represent the kinetic spectra for the simula- 


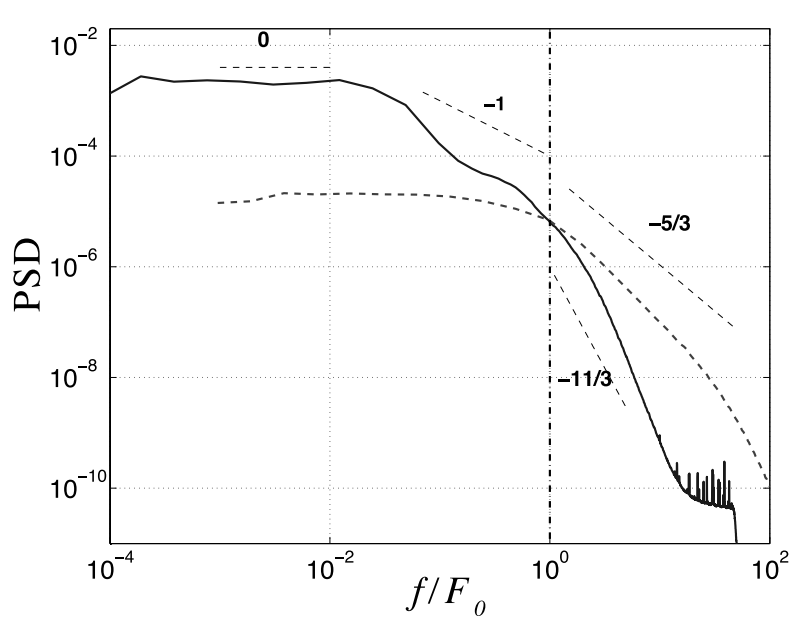

FIG. 4. Experiments of MHD induction in VKG flow. Power spectrum of the axial velocity (light gray dashed line) and of the induced axial magnetic field in VKG experiment with a weak imposed transverse magnetic field. Frequency axis has been normalized by the forcing frequency $F_{0}$ taken as the rotation rate of the impellers $\Omega(10 \mathrm{~Hz}$ for the shown experiment). The global energy level of the spectra has been arbitrarily set to make them coincide for $f=F_{0}$. Several slopes are shown as references.

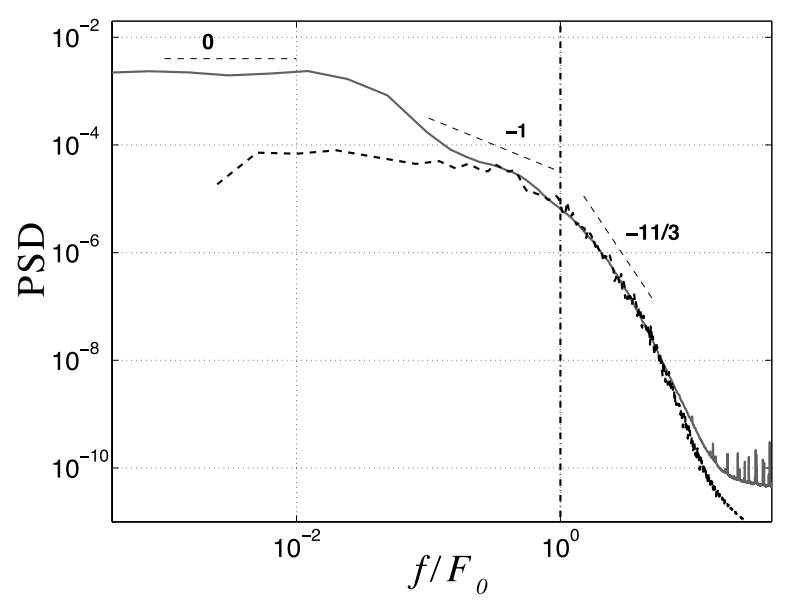

FIG. 5. Experiments vs. simulations (induction case): comparison of the spectra of the induced magnetic field in figures 3 (simulation) and 4 (experiment). The total energy level has been arbitrarily set to make both spectra coincide at $f=F_{0}$. Slopes are shown as references. Measurements from the simulation and the experiment agree remarkably well except for very low frequencies.

tion and the experiment respectively. A relatively clear inertial range of time scales, consistent with a classical $f^{-5 / 3}$ Kolmogorov spectrum is present, but no significant long term dynamics is observed at low frequencies, and the spectra flatten rapidly below the forcing frequency $F_{0}$ (in the experiment $F_{0}$ is taken as $\Omega$, the rotation rate of the impellers, while in the simulation $F_{0}$ is determined as $u_{r m s} / L$, where $L=2 \pi / k_{0}=\pi$ is the forcing scale and $u_{r m s}$ is the root mean square of the turbulent velocity fluctuations).

The absence of long-term dynamics is in agreement with previous results indicating isotropic and homogeneous hydrodynamic turbulence does not display $1 / f$ noise [6]. However, and unlike the results in Ref. [6], when in the numerical simulations the time series of the amplitude of individual Fourier modes are considered, we find that modes in the $k=1$ shell do have $1 / f$ spectra (the observed spectrum is actually steeper than $1 / f$, see the bottom panel of Fig. (3). Interestingly, it can be noted that the modes in this Fourier shell break down the symmetries of the TG flow and, as an example, are responsible for large-scale fluctuations in the $z$ position of the shear-layer between the two Taylor-Green vortices. Indeed, $1 / f$ spectra are only observed in modes that break down this symmetry. The spectrum of the temporal fluctuations of small-scale modes (large wave-numbers) does not exhibit any long-term dynamics, as it can be seen in Fig. 3 (bottom). However, at least at the Reynolds number considered in the simulation, the modes that break down the symmetry do not have enough energy to give rise to a clear long-term behavior when all Fourier modes are integrated to obtain the velocity at one of the measurement points (as shown in the top panel of Fig. 3).

As already mentioned, when modes with $1 / f$ behavior are not the most energetic, $1 / f$ behavior is not observed in global quantities. However, as soon as a physical effect makes these modes dominant, $1 / f$ noise arises. This can be observed for instance by considering the evolution of the small imposed magnetic field with low magnetic Prandtl number and low interaction parameter $(N \ll 1)$. Condition $N \ll 1$ ensures that the magnetic field passively traces the carrier velocity field, while condition $\mathrm{Pm} \ll 1$ imposes a large scale separation between magnetic and velocity field, so that only large scales of the velocity field are traced by the magnetic field. Figure 3 (top) also shows the spectrum of the axial induced magnetic field, in the presence of a small applied transverse field, from the numerical simulation (with $\mathrm{Pm}=0.05$ and $N=4 \times 10^{-5}$ ). A short but still visible $1 / f$ regime appears for frequencies below the forcing frequency $F_{0}$. As previously noted, this regime is not visible for the velocity spectrum. For the comparison, the global energy level of the kinetic and magnetic spectra has been set so as to make them coincide at $f=F_{0}$.

Similarly, Fig. 目 shows the spectrum of the velocity measured in VKG experiment with a Vivès probe compared to the spectrum of the induced axial magnetic field (measured at point 1) when a weak passive transverse magnetic excitation $B_{0 x}$ is imposed (note that high frequency resolution of the Vivès probe is limited by magnetic diffusion at the probe size, which is responsible for the cut-off observed here for frequencies $f \gtrsim 200 \mathrm{~Hz}[20]$ ]). For the comparison, the global energy level of the kinetic and magnetic spectra has been set so as to make them coincide at $f=F_{0}$. Contrary to the kinetic spectrum, 

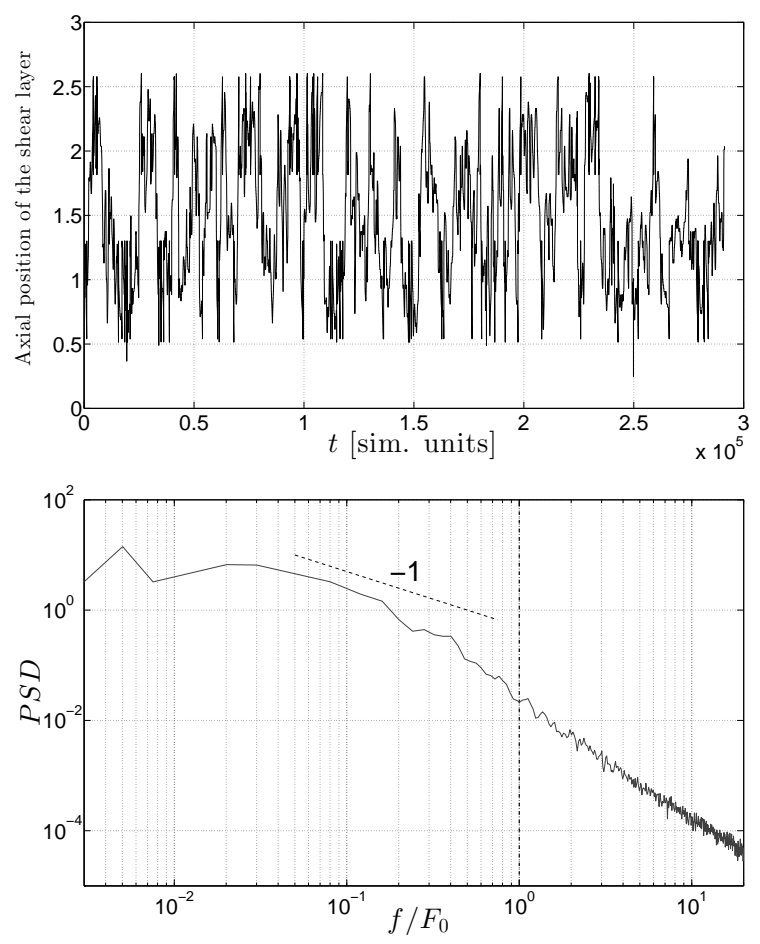

FIG. 6. Numerical simulations. Top: position of the shear layer as a function of time (in simulation units). Bottom: power spectrum of the signal in the top figure. A slope of -1 is indicated as a reference.

the magnetic one shows an important long term dynamics below $F_{0}$, that, at the light of the previous numerical observations, shall be attributed to the large scale modes of the kinetic field naturally traced by the magnetic field in such a low magnetic Prandtl number regime. In other words, the passive magnetic field at very low Pm acts as a low-pass filter that only senses the velocity field at very large scales.

To push further the comparison between the experiment and the simulation, Fig. 5 shows the magnetic spectra from the simulation and the experiment in Figs. 3 and 4 (axial component of the induced magnetic field measured at point 1 in the simulation and at point 1 in VKG, which corresponds to the closest available geometries between experiments and simulations). The total energies have been rescaled so that the spectra in the numerics and in the experiment are equal for $f=F_{0}$. We recall that the frequency axis has been normalized by the corresponding forcing frequency $F_{0}: F_{0}=u_{r m s} / \pi$ for the simulation, and $F_{0}=\Omega$ the rotation rate of the disks for the experiment. Interestingly, this scaling was found to give the best collapse between numerics and experiment. As it can be seen in the figure, the agreement is indeed remarkable, except for the very low frequencies which are enhanced in the experiment. The slow $1 / f$ dynamics for frequencies $f \lesssim F_{0}$ is in particular observed in both studies. It is also interesting to note that this agreement for the dynamical features of the induction processes is
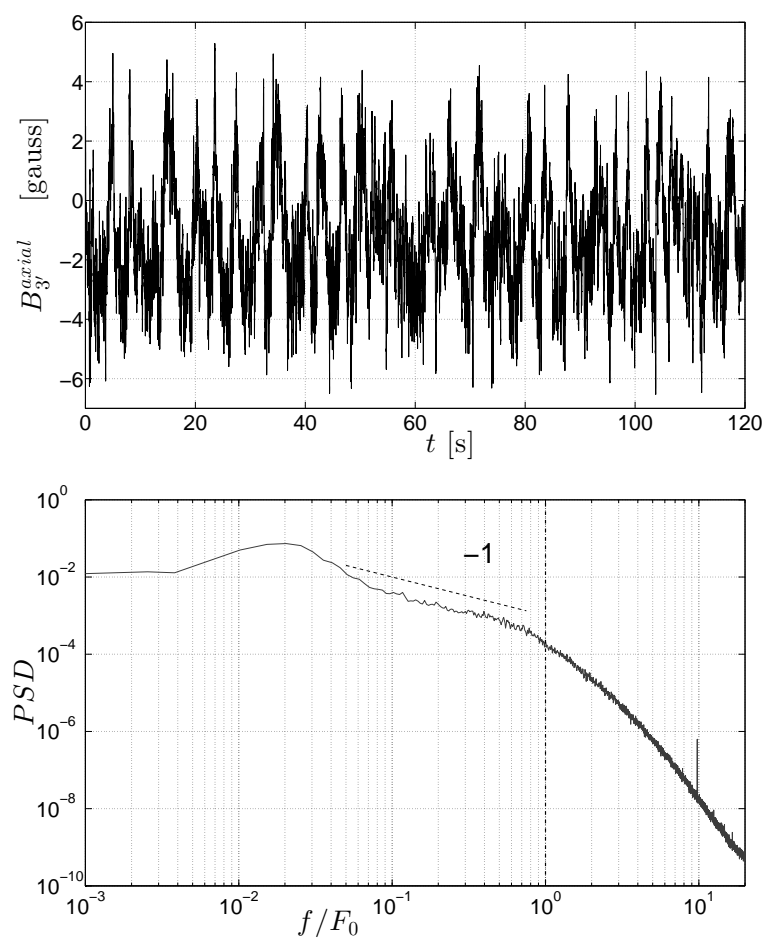

FIG. 7. VKG experiment. Top: proxy of the evolution of the shear layer as a function of time, as indicated by the evolution of the axial induced magnetic field measured at point 3'. By $\mathcal{R}_{\pi}$ symmetry around the $O x$ axis, this component of induced field must be zero. However, it is visible from the measurements that it fluctuates between a high level state (of order 4 gauss) and a low level state (of order -4 gauss). We interpret these fluctuations as a signature of the instantaneous breaking of the $\mathcal{R}_{\pi}$ symmetry due to the motion of the shear layer. Bottom: power spectrum of the signal in the top figure. A slope of -1 is indicated as a reference.

observed even if the dominant induction mechanisms are different in the numerics and the experiment. It has indeed been shown in the experiment that the mean axial component induced at the location of the measurement point 1 when a transverse field $B_{0 x}$ is applied results from a boundary condition effect due to conductivity difference between the wall of the experiment and the fluid, combined to the presence of local flow vorticity in the vicinity of the boundary [26] (BC mechanism). Such a mechanism cannot be present in the numerics where the entire medium has a homogenous conductivity. This may explain the difference between the simulation and the experiment at very low frequencies, which in the experiment are dominated by this very specific average induction processes.

However, in both cases the fluctuations of induction processes do trace back the fluctuations of the flow. The long-term effects at $f \lesssim F_{0}$ for intermediate frequencies must therefore be associated with fluctuations in the velocity field that are present in both the experiments and simulations. In other words, the remarkable collapse 

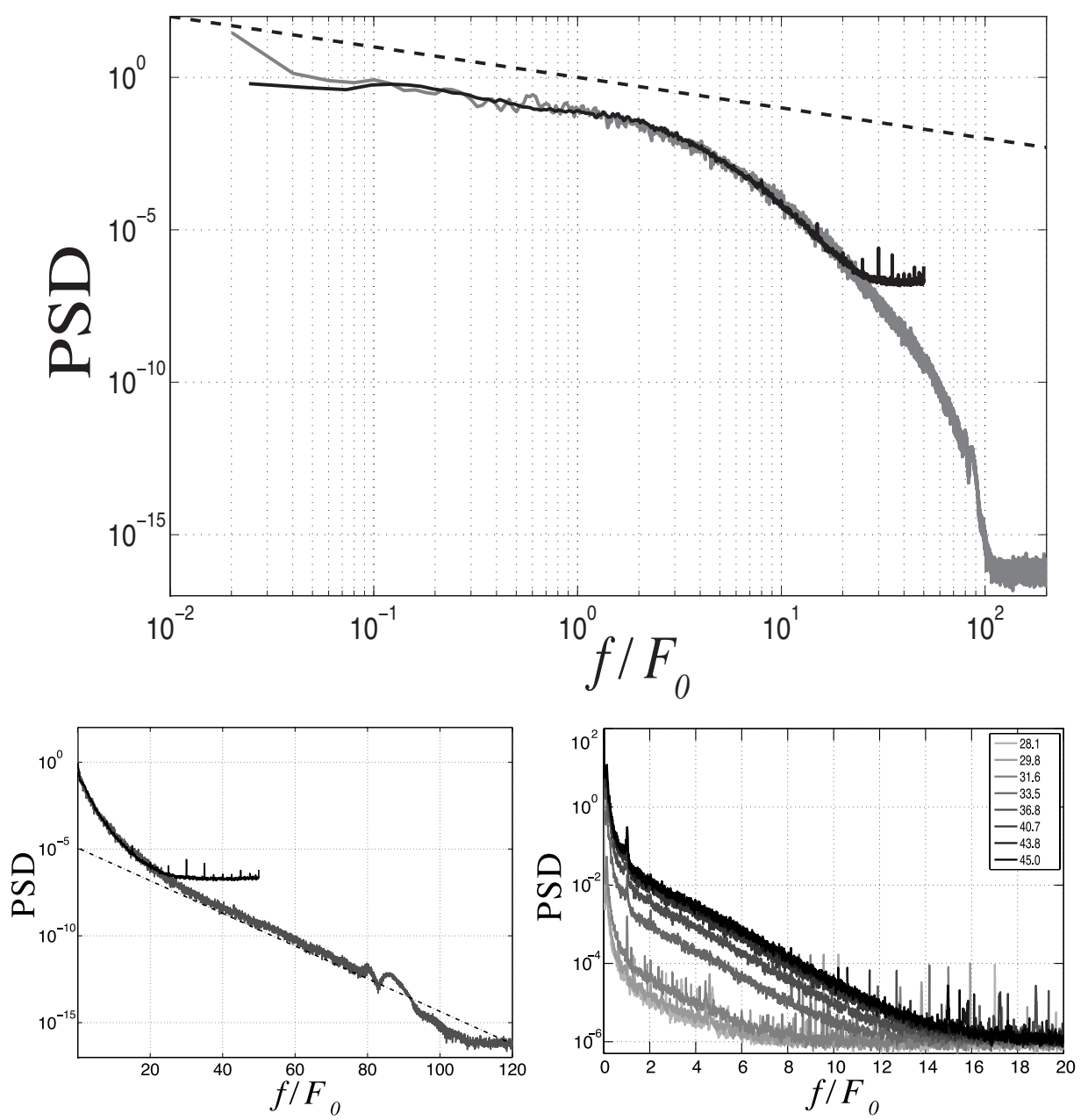

FIG. 8. Experiments vs. simulations in the dynamo case. Top: power spectra of the self sustained magnetic field in the VKS experiment (black) and in the simulation (gray). A slope of -1 is indicated as a reference. Bottom left: semilog plot of top figure. Bottom right: semilog plot of magnetic spectra measured at point 3 in VKS (from Ref. [13]). The different curves correspond to different magnetic Reynolds numbers as indicated in the legend, with increasing Rm from light gray to black curves.

of numerical and experimental spectra for moderate and high frequencies indicates that the magnetic field fluctuations at these frequencies are tracing fluctuations of the velocity field with a common origin.

The numerical observation that $1 / f$ is essentially related to large scale hydrodynamic modes breaking the symmetries of the TG flow, and the experimental observation of a $1 / f$ regime in a configuration where one of the sources of induction is related to radial vorticity in the mid-plane, both point toward an important role (for intermediate frequencies) of the fluctuations of the strong shear layer in the mid-plane of the von Kármán flow. This shear layer is indeed known to undergo strong large scale and long term fluctuations in experimental von Kármán flows [2, 18, 27]. These fluctuations are also observed in the simulation, and are shown in Fig. 6. which represents the time series of the position of the shear layer as a function of time, and its power spectrum in the numerics. To identify the position of the shear layer in the simulations, a low-pass filtered and averaged (in $x$ and $y$ ) velocity field was computed in the TG cells from the Fourier coefficients of the velocity, leaving mean profiles for the azimutal and $z$ component of the velocity field that depend only on $z$ (see Fig. 22).

In the VKG experiments, a proxy of the evolution of the shear layer can be obtained from measurements of magnetic induction. A particularly clear tracing of the shear layer is obtained when measuring the induced axial magnetic field $B_{x}^{i}$ at a location on the horizontal $O x$ axis (point 3 ' in Fig. 1) for an applied transverse magnetic field $B_{0 x}$. Simple symmetry considerations impose indeed the induced axial magnetic field measured at any point of the $O x$ axis for an applied transverse field $B_{0 x}$ to be zero on average [28]. Figure 7 (top) shows the corresponding time series which clearly shows that the measured signal does fluctuate around zero, but that it explores induction regimes with symmetric polarities which can be attributed to slow symmetry breaking fluctuations 

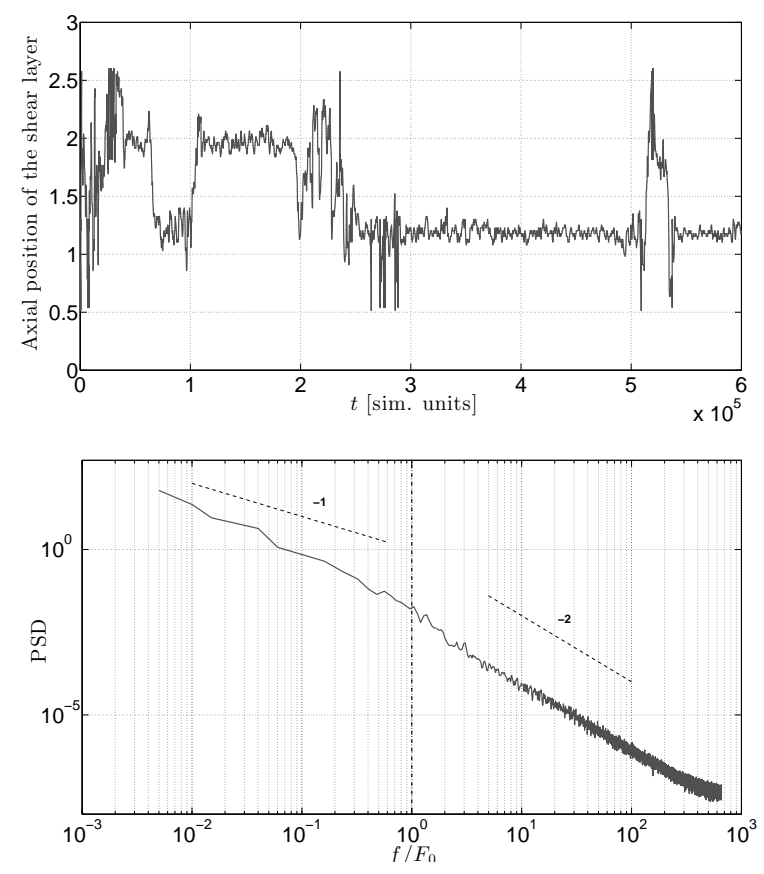

FIG. 9. Position of the shear layer as a function of time in the numerical simulations of dynamo action, and power spectrum of the signal. A slope of -1 is indicated as a reference.

of the shear layer respective to its equilibrium position in the mid-plane [28]. Figure 7 (bottom) shows the corresponding spectrum which does exhibit a clear long-term $1 / f$ regime.

\section{MAGNETOHYDRODYNAMIC DYNAMO}

We now turn to the case of MHD dynamo and compare dynamical fluctuations observed in the VKS experiment and numerical results from the present TG simulations. Though parameters are not really comparable (in particular the Prandtl number differs by orders of magnitude as it is of the order of $6 \times 10^{-6}$ in VKS while it is 0.5 in the simulation), experimental and numerical spectra of magnetic fluctuations show a remarkable good agreement, with in particular a wide $1 / f$ range as shown in Fig. 8 (top), where we have plotted the magnetic spectra measured in the bulk at point 1 in VKS and in the simulation. As in the hydrodynamic case, this long-term memory is associated with the evolution of the large-scale modes. However, the magnetic field now appears to develop a $1 / f$ regime over a much wider range of scales (almost 2 decades) than in the passively advected case. This indicates that the dynamo field develops its own long-term dynamics, which increases the correlation time scale as the magnetic field produced by the dynamo is spatially correlated at the largest available scale in the box. As a result, the range of time scales for which longterm memory is observed is substantially increased in the case of the self-sustained dynamo, which can also be observed in the evolution of the position of the shear layer with time (see Fig. 9). Interestingly, we also note that the remarkable collapse of the experimental and numerical spectra in Fig. 8 (top) is obtained when the frequency in the simulation is normalized by $F_{0}=u_{r m s} / 2 L$, with $L=\pi$ the forcing scale of the TG simulation and not by $F_{0}=u_{r m s} / L$ as was the case for the passively advected magnetic field previously discussed. This is related to the large scale correlation of the dynamo field, which is now correlated over the entire simulation box which is twice the forcing scale.

The existence of $1 / f$ slow dynamics of magnetic fluctuations, related to the large scale nature of magnetic field, seems to be a robust behavior of MHD large-scale dynamos. It is consistently observed in VKS experiment and TG simulations, which have similar flow geometries, but different boundary conditions, MHD parameters (mostly in terms of Prandtl numbers), and which have very likely different dynamo generating mechanisms (VKS generates an axial dipole and is very sensitive to electromagnetic boundary conditions, while TG generates an equatorial dipole and operates in a homogeneous medium). A slow dynamics $1 / f$ regime has also been reported in the Karslruhe dynamo experiment, which has a different geometry, different fluctuation levels, and different dynamo mechanism. In Ref. [14] it is argued from the results of a large number of numerical simulations that in systems that develop inverse cascades (as, e.g., helical MHD turbulence) the development of $1 / f$ noise and long-term memory is more robust, in the sense that it is independent of details of the flow geometry and forcing. The present results are in good agreement with these arguments, and confirms in experiments the previous numerical results.

An interesting observation can also be done regarding the dissipative regime of the high frequency magnetic spectra. As observed in Fig. 8(top), this regime is not resolved in the experimental data, due to limitations in dynamical resolution of the magnetic measurements when performed in the bulk of the flow. However, the dissipation behavior can be better resolved from measurements in the outer layer of sodium, in the vicinity of the copper shell (for instance at point 3 in the VKS sketch in Fig. 10), where no flow is present and magnetic dynamics results essentially from diffusion processes of the turbulent magnetic fluctuations in the bulk. Such measurements show that the far dissipation spectrum of magnetic dynamo field decreases exponentially with the frequency $f$ (as an example, the bottom right panel of Fig. 8) reproduces results from Fig. 16(c) in [13]). Though we do not have an explanation for such an exponential spectral regime, we interestingly find that the same exponential behavior is captured by the simulation as shown in Fig. 8 (bottom left). TG simulations therefore are able to reproduce a large number of temporal spectral properties of turbulent magnetic fluctuations as measured in the VKS experiment. 


\section{CONCLUSIONS}

We presented a comparison of time series of pointwise measurements of the velocity and of the magnetic field, both from direct numerical simulations and from experiments of turbulence in conducting flows. Two configurations were considered: (1) A case with a weak externally imposed magnetic field, in which induction generated by fluid motions can be used as a tracer of the velocity field (and mostly of the large scale modes of the velocity field, as the magnetic Prandtl number considered in that case is small). And (2) a case with self-sustained magnetic fields. The flow in the experiment corresponds to a von Kármán swirling flow between two counter-rotating impellers in a cylindrical vessel, while in the simulations the flow is generated in a square box using periodic TaylorGreen forcing.

While experiments excel at providing time statistics of field fluctuations with limited spatial information of the flow geometry, numerical simulations tend to be performed at high resolution for short times, thus providing substantial amounts of spatial information with little time statistics. In the present study a different approach was used, considering low resolution simulations but extended for very long times, so comparisons between experimental and numerical data can be done on the same grounds.

Good general agreement was found between spectral properties in simulations and experiments, both in the hydrodynamic (weak imposed magnetic field) and dynamo cases. Intermediate and high frequencies spectra from the experiments were found to be well reproduced by the simulations. In particular, evidence of long-term memory and $1 / f$ noise at intermediate frequencies was found in both experiments and simulations. In the hydrodynamic case, low frequency magnetic spectra were found to deviate between the experiment and the simulation. This can be attributed to different induction processes operating in the bounded experiment and in the periodic simulations.

In the hydrodynamic regime, $1 / f$ noise in the kinetic spectrum is a signature of the slow fluctuations of the largest structures of the flow, and particularly of the mid-plane shear-layer. As a consequence, this is only observed in the simulation when small wave numbers are selected. This selection is naturally operated when considering magnetic fluctuations of a passively advected magnetic field in low Prandtl number situations, which are indeed found to exhibit $1 / f$ behavior.

In the dynamo case, $1 / f$ noise of magnetic field fluctuations is enhanced over a wider range of scales, that is attributable to the intrinsic large scale nature of the generated field. The remarkable collapse of simulations (at $\mathrm{Pm}=0.5)$ and experiments (at $\mathrm{Pm} \approx 10^{-} 5$ ) indicates that this may be a fundamental and robust property of large-scale MHD dynamos where slow fluctuations (both kinematic and magnetic) appear when the magnetic and velocity field become intimately coupled. This last conclusion may be particular useful to extrapolate dynamical behaviors from simulations at $\mathrm{Pm} \approx 1$ (a condition computationally favorable) to more realistic dynamos (natural or experimental) at small Prandtl regimes (which are computationally expensive to simulate). A particular example where this may be relevant is the case of dynamo reversals, which take place on very long time scales and which cannot be studied in simulations at low magnetic Prandtl number.

\section{ACKNOWLEDGMENTS}

The authors acknowledge support from grant ECOS A08U02. PDM and PD acknowledge support from grants No. PIP 11220090100825, UBACYT 20020110200359, and PICT 2011-1529 and 2011-1626. The french authors acknowledge their colleagues of the VKS team with whom experimental data have been obtained. Their work was supported by ANR 08-0039-02.
[1] A. de la Torre and J. Burguete, Physical Review Letters 99, 054101 (2007).

[2] E. Crespo Del Arco, J. J. Sanchez-Alvarez, E. Serre, A. De La Torre, and J. Burguete, Geophysical and Astrophysical Fluid Dynamics 103, 163 (2009).

[3] E. W. Montroll and M. F. Shlesinger, Proceedings of the National Academy of Sciences of the United States of America 79, 3380 (1982).

[4] S. Machlup, Sixth International Conference on Noise in Physical Systems pp. 157-160 (1981).

[5] Y. Ponty, H. Politano, and J. Pinton, Physical Review Letters 92, 144503 (2004).

[6] P. Dmitruk and W. H. Matthaeus, Physical Review E 76, 036305 (2007).

[7] M. Goldstein, D. Roberts, and W. Matthaeus, Annual Review of Astronomy and Astrophysics 33, 283 (1995).
[8] W. Matthaeus and M. Goldstein, Physical Review Letters $\mathbf{5 7}, 495$ (1986).

[9] W. H. Matthaeus, B. Breech, P. Dmitruk, A. Bemporad, G. Poletto, M. Velli, and M. Romoli, Astrophysical Journal 657, L121 (2007).

[10] V. Carbone, L. Sorriso-Valvo, A. Vecchio, F. Lepreti, P. Veltri, P. Harabaglia, and I. Guerra, Physical Review Letters 96, 128501 (2006).

[11] L. Ziegler and C. Constable, Earth and Planetary Science Letters 312, 300 (2011).

[12] C. Constable and C. Johnson, Physics of the Earth and Planetary Interiors 153, 61 (2005).

[13] R. Monchaux, M. Berhanu, S. Aumaitre, A. Chiffaudel, F. Daviaud, B. Dubrulle, F. Ravelet, S. Fauve, N. Mordant, F. Petrelis, et al., PHYSICS OF FLUIDS 21 (2009), ISSN 1070-6631. 
[14] P. Dmitruk, P. D. Mininni, A. Pouquet, S. Servidio, and W. H. Matthaeus, Physical Review E 83, 066318 (2011).

[15] P. Mininni, Ann. Rev. Fluid Mech. 43, 377397 (2011).

[16] R. Volk, P. Odier, and J.-F. Pinton, Physics of Fluids 18, 085105 (2006).

[17] G. Verhille, N. Plihon, M. Bourgoin, P. Odier, and J.-F. Pinton, Space Science Reviews 152, 543 (2010), ISSN 0038-6308, URL http://www.springerlink.com/index/10.1007/s11214-

[18] M. López-Caballero and J. Burguete, Physical Review Letters 110, 124501 (2013).

[19] G. Verhille, R. Khalilov, P. Frick, N. Plihon, and J.-F. Pinton, Journal of Fluid Mechanics 693, 243 (2012).

[20] V. Noskov, R. Stepanov, S. Denisov, P. Frick, G. Verhille, N. Plihon, and J.-F. Pinton, Physics of Fluids 21, 045108 (2009).

[21] Y. Ponty, P. Mininni, D. Montgomery, J. Pinton, H. Politano, and A. Pouquet, Physical Review Letters 94, 164502 (2005).
[22] P. Mininni, Y. Ponty, D. Montgomery, J. Pinton, H. Politano, and A. Pouquet, Astrophysical Journal 626, 853 (2005).

[23] D. Gomez, P. Mininni, and P. Dmitruk, PHYSICA SCRIPTA T116, 123 (2005), ISSN 0031-8949, International Workshop on Theoretical Plasma Physics, Trieste, ITALY, JUL 05-16, 2004.

[24] D. Gomez, P. Mininni, and P. Dmitruk, Fundamentals of 09-9548pace Environment Science 35, 899 (2005).

[25] P. D. Mininni, D. Rosenberg, R. Reddy, and A. Pouquet, Parallel Computing 37, 316 (2011).

[26] M. Bourgoin, P. Odier, J.-F. Pinton, and Y. Ricard, Physics of Fluids 16, 2529 (2004).

[27] F. Ravelet, A. Chiffaudel, and F. Daviaud, Journal of Fluid Mechanics 601, 339 (2008).

[28] M. Bourgoin, R. Volk, P. Frick, S. Khripchenko, P. Odier, and J.-F. Pinton, Magnetohydrodynamics 40, 13 (2004). 